\title{
Cuantificación de gases de efecto invernadero en la Sede de Occidente de la Universidad de Costa Rica
}

\section{Quantification of Greenhouse Gases (GHG) generated in the University of Costa Rica-West Seat}

\author{
María José Chassoul A. ${ }^{1}$ \\ Rolando Marín L. ${ }^{2}$ \\ María Alejandra Morales C. ${ }^{3}$ \\ María Fernanda Fallas M. ${ }^{4}$ \\ Universidad de Costa Rica, Costa Rica
}

\begin{abstract}
Resumen. La Sede de Occidente de la Universidad de Costa Rica se ubica en San Ramón de Alajuela. Fue fundada en abril de 1968 y actualmente cuenta con una población de aproximadamente 3100 estudiantes y 911 funcionarios, los cuales se distribuyen entre el recinto de San Ramón y el de Grecia. Con el propósito de alinearse con el compromiso de la Universidad de Costa Rica de ser Carbono Neutro en el 2020, la Sede decidió cuantificar los Gases de Efecto Invernadero (GEI) generados por las actividades realizadas con el objetivo de establecer una estrategia de mitigación y poder disminuir el impacto ambiental causado. Para la cuantificación. primero se identificaron las diferentes fuentes emisoras de cada instalación física; luego se realizó la recolección de datos y, por último, se calculó la cantidad de toneladas de carbono equivalente emitidas de acuerdo a los factores de emisión establecidos por el Instituto Meteorológico Nacional. Esto permitió determinar que la Sede genera 267,35 toneladas de CO2-e, de las cuales 93\% corresponde al recinto de San Ramón y 7\% al recinto de Grecia.
\end{abstract}

Palabras clave. Gases de Efecto Invernadero, Sede de Occidente, Universidad de Costa Rica

\begin{abstract}
W. The West Seat of the University of Costa Rica is located in San Ramón, Alajuela. It was founded in April 1968 and currently houses a population of approximately 3100 students and 911 staff members, distributed between the facilities in San Ramon and Grecia. To align itself with the University's commitment to be Carbon Neutral by 2020, the West Seat decided to quantify the Greenhouse Gases GHG generated by its activities and then establish a mitigation strategy to lessen the impact caused. For the quantification, first the different emission sources were identified in the facilities, then data collection was performed, and finally, the number of equivalent tons of carbon emitted, according to the emission factors established by the Instituto Metereológico Nacional [National Meteorology Institute], was calculated. This made it possible to determine that the West Seat generates 267.35 tons of CO2-e, of which 93\% corresponds to the San Ramon site and 7\% to the Grecia site.
\end{abstract}

Keywords. Greenhouse Gases, Sede de Occidente, Universidad de Costa Rica

${ }^{1}$ MaríaJoséChassoulA.Profesora einvestigadoradelacarrera deIngeniería Industrial,SededeOccidente,Universidad deCosta Rica.maria.chassoul@ucr.ac.cr ${ }^{2}$ Rolando Marín L. Profesor e investigador de la carrera de Ingeniería Industrial, Sede de Occidente, Universidad de Costa Rica. Dirección electrónica: rolando.marin@ucr.ac.cr

${ }^{3}$ María Alejandra Morales C. Ingeniera industrial de la Universidad de Costa Rica. Dirección electrónica: alejandramorales147@gmail.com

${ }^{4}$ María Fernanda Fallas M. Ingeniera industrial de la Universidad de Costa Rica. Dirección electrónica: mafer_8808@hotmail.com 


\section{Introducción}

Los Gases de Efecto Invernadero (GEI) son un "componente atmosférico, tanto natural como antropogénico, que absorbe y emite radiación a longitudes de onda específicas dentro del espectro de radiación infrarroja emitida por la superficie de la Tierra, la atmósfera y las nubes" (Instituto de Normas Técnicas de Costa Rica [INTECO], 2013, p. 9). Sin los GEI, la Tierra sería demasiado fría para albergar vida (Intergovernamental Panel on Climate Change [IPCC], 2001), sin embargo, el aumento de estos gases como producto de las actividades antropogénicas están ocasionando el cambio climático. El cual es definido por la Convención Marco de las Naciones Unidas sobre el Cambio Climático como "cambio de clima atribuido directa o indirectamente a la actividad humana que altera la composición de la atmósfera mundial y que se suma a la variabilidad natural del clima observada durante periodos de tiempo comparables" (Naciones Unidas, 1992, p. 6).

Como parte de las iniciativas generadas para combatir el cambio climático, se estableció la carbono neutralidad (C-N), la cual, según la Universidad EARTH (uno de los promotores de la carbono neutralidad en Costa Rica), se entiende como "el balance entre la cuantificación de las emisiones y las acciones de reducción y remoción/compensación de gases de efecto invernadero de una organización en un periodo verificable" (Universidad EARTH, 2014, parr. 1). Es decir, el balance entre lo que se emite y lo que se hace para reducir estas emisiones. Según la norma nacional INTE 12-01-06:2011, ser carbono neutro "significa que las emisiones netas asociadas con un producto o las actividades de la organización sean iguales a cero". Esto se logra "cuando a través de un proceso transparente de medición de las emisiones (e), el resultado del cálculo neto de las emisiones menos las reducciones y/o ๑sic® remociones internas (r), menos la compensación (c) es igual a cero." (INTECO, 2013, p.8).

Uno de los elementos asociados a la ecuación de la carbono neutralidad es la remoción o mitigación de las emisiones de los GEI. lo cual se entiende dentro de la Estrategia Nacional de Cambio Climático como: "intervención antropogénica para reducir las fuentes o mejorar los sumideros de gases con efecto invernadero" (Ministerio de Ambiente, Energía y Telecomunicaciones [MINAET], 2009, p. 100). O sea, es la intervención que el ser humano realiza para reducir las emisiones de los GEI o mejorar su captación.

El inventario de los GEI permite identificar la cantidad de gases que se emiten en una organización y aquellas actividades que causan dichas emisiones. Los GEI que deben ser considerados en este inventario, corresponden a los que establece el Protocolo de Gases de Efecto Invernadero y que han sido listados en el Protocolo de Kyoto: Dióxido de carbono $\left(\mathrm{CO}_{2}\right)$, Metano $\left(\mathrm{CH}_{4}\right)$, Óxido nitroso $\left(\mathrm{N}_{2} \mathrm{O}\right)$, Hidrofluorocarbonos (HFCs), Perfluorocarbonos (PFCs), Hexafluoruro de azufre $\left(\mathrm{SF}_{6}\right)$ (Protocolo de Kyoto, 1998). Estos gases son considerados también por el Instituto Meteorológico Nacional (IMN), el cual incluye además Monóxido de carbono (CO), Óxido de nitrógeno $\left(\mathrm{NO}_{\mathrm{X}}\right)$, Hidrocarburos volátiles diferentes del metano (NMVOC) y Dióxido de azufre $\left(\mathrm{SO}_{2}\right)$ (Instituto Metereológico Nacional, 2014).

La norma nacional para demostrar la C Neutralidad INTE 12-01-06:2011 dicta que la unidad para realizar el inventario de GEI debe ser el $\mathrm{CO}_{2}$-equivalente $\left(\mathrm{CO}_{2}\right.$-e), la cual se define de la siguiente manera:

Unidad universal de medida que indica el potencial de calentamiento global (PCG), de cada uno de los seis gases [de] efecto invernadero, expresado en términos del PCG de una unidad de dióxido de carbono. Se utiliza para evaluar la liberación (o el evitar la liberación), de diferentes gases efecto invernadero contra un co- 
mún denominador (World Business Council for Sustainable Development, World Resource Institute y Secretaria de Medio Ambiente y Recursos Naturales, 2005).

Como parte de la metodología propuesta por esta norma, se realiza una clasificación de las emisiones en directas e indirectas. Las emisiones directas representan aquellas "emisiones de GEI provenientes de fuentes de GEI que pertenecen o son controladas por la organización" (INTECO, 2006, p. 8). Las indirectas se encuentran divididas en dos tipos: las emisiones indirectas de GEI por energía, que corresponden a la "emisión de GEI que proviene de la generación de electricidad, calor o vapor de origen externo consumidos por la organización" (INTECO, 2006, p. 8) y las otras emisiones indirectas de GEI referentes a la emisión de GEI "consecuencia de las actividades de la organización, pero que se originan en fuentes GEI que pertenecen o son controladas por otras organizaciones" (INTECO, 2006, p. 8).

Durante la Cumbre de las Naciones Unidas sobre Cambio Climático en 2009, el gobierno de Costa Rica adquirió el compromiso de alcanzar para el 2021 la carbono neutralidad, lo que responsabiliza a los habitantes del país y a las entidades públicas y privadas a sumarse a esta meta ya próxima. En el caso específico de la Universidad de Costa Rica (UCR), el 12 de mayo del 2011, durante la sesión 5539 el Consejo Universitario aprobó la propuesta de ser Carbono-Neutral (Universidad de Costa Rica [UCR], 2011). Por este motivo se creó la Comisión institucional de Carbono Neutralidad la cual planteó como meta lograr en el año 2020 la carbono neutralidad en todas las sedes, los recintos, los centros y los institutos de investigación. Con el propósito de alinearse con el compromiso de la Universidad, se planteó esta investigación en la Sede de Occidente entre los años 2015 y 2016, por medio de un proyecto de tesis de licenciatura en la carrera de ingeniería industrial (Morales y Fallas, 2016). El objetivo fue cuantificar los GEI generados por las actividades realizadas en la Sede, para luego establecer una estrategia de mitigación y poder disminuir el impacto ambiental causado por la misma, en aras de alcanzar la Carbono Neutralidad en la institución.

\section{Metodología}

El proyecto se realizó en la Sede de Occidente de la Universidad de Costa Rica. Esta Sede fue fundada en abril de 1968 y posee una población de aproximadamente 3100 estudiantes y 911 funcionarios. Está conformada por el recinto de San Ramón y el de Grecia, y se encuentra constituida por cinco coordinaciones: Administración, Acción Social, Investigación, Vida Estudiantil y Docencia; además tiene bajo su administración el Conservatorio de Música de Occidente, la Reserva Biológica Alberto Manuel Brenes, el Museo Regional de San Ramón, el Centro Infantil Laboratorio Ermelinda Mora y las residencias estudiantiles. Asimismo, ofrece 31 carreras distribuidas en cuatro departamentos: Ciencias Naturales, Ciencias Sociales, Ciencias de la Educación y Filosofía, Artes y Letras (UCR, 2017).

Para cuantificar la cantidad de Gases de Efecto Invernadero (GEI) de la Sede, primero se identificó en cada instalación física los tipos de fuentes emisoras (tabla 1), luego se realizó la recolección de datos y por último se calculó la cantidad de toneladas de carbono emitidas por cada una de las fuentes, de acuerdo con los factores de emisión establecidos por el Instituto Meteorológico Nacional (IMN). Es importante señalar que este fue un proceso arduo, en el cual se debió hacer prueba de adaptabilidad y creatividad en la recolección de datos, dado que se presentaron serios problemas para recabar la información, debido sobre todo a la falta sistemática de registros o de un protocolo para su ordenamiento.

Para la recolección de datos referentes a cada una de las fuentes se utilizó como año base de 
medición el 2014 y se utilizó la metodología descrita a continuación:

\section{Combustible}

Para determinar el consumo de combustible fue necesario realizar la digitación de las facturas correspondientes al año base. Se recolectaron los datos referentes a cantidad, tipo de combustible adquirido, precio y placa del vehículo al que se le recargó el combustible.

\section{Excretas}

El IMN establece un factor de emisión por excretas humanas de 4,38 $\mathrm{kg} \mathrm{CH} 4 /$ persona/año en el caso del uso de tanques sépticos, y de 2,63 $\mathrm{CH} 4 /$ persona/año para una laguna de tratamiento por persona los 365 días del año, las 24 horas del día. Debido a que en el Recinto de San Ramón una parte de las aguas residuales son tratadas por la planta y la otra con tanques sépticos $\mathrm{y}$, adicionalmente, las personas no pasan las 24 horas del día en las instalaciones, fue necesario realizar un ajuste por tipo de usuario, como se describe a continuación. En las otras instalaciones, todas las excretas son tratadas por medio de tanque séptico.
En el caso de los administrativos, se conoce el tiempo que ellos permanecen en las instalaciones y la cantidad de personas. Entonces, se calculó el tiempo total durante el año que este personal permanece en la Sede. Para los docentes, se tomó en cuenta la cantidad de cursos promedio por profesor, la duración y las horas oficina que realizan (estas últimas contemplan las horas en las que se encuentran en la oficina, horas consulta e investigación). Una vez obtenido el dato, se ajustó el factor igual que en el caso de los administrativos.

En cuanto a los estudiantes, se tomó en cuenta la cantidad de cursos promedio por estudiante y su duración. Para determinar el tiempo adicional que permanecen en las instalaciones, se utilizaron los datos obtenidos por un estudio realizado previamente por estudiantes de Ingeniería Industrial, en el curso de Localización y Distribución de Instalaciones, el cual brinda el tiempo promedio que un estudiante permanece en la Sede para estudio y otros fines (Rodríguez, Campos, Ramírez, Mora y Ramírez, 2014)

Una vez obtenida la información ajustada para cada caso, en el Recinto de San Ramón se realizó un análisis de recurrencia en los diferentes edi-

Tabla 1

Fuente emisoras de GEI por instalación física de la Sede de Occidente

\begin{tabular}{ccccccc}
\hline \multirow{2}{*}{ Instalación } & \multicolumn{5}{c}{ Fuente emisora } \\
\cline { 2 - 7 } & Combustibles & Electricidad & $\begin{array}{c}\text { Excretas } \\
\text { Humanas }\end{array}$ & $\begin{array}{c}\text { Residuos } \\
\text { sólidos }\end{array}$ & $\begin{array}{c}\text { A/C y refrige- } \\
\text { rantes }\end{array}$ & Gas \\
\hline Recinto San Ramón & $\mathrm{X}$ & $\mathrm{X}$ & $\mathrm{X}$ & $\mathrm{X}$ & $\mathrm{X}$ & $\mathrm{X}$ \\
Recinto Grecia & $\mathrm{X}$ & $\mathrm{X}$ & $\mathrm{X}$ & $\mathrm{X}$ & $\mathrm{X}$ & $\mathrm{X}$ \\
Residencias San Ramón & $\mathrm{N} / \mathrm{A}$ & $\mathrm{X}$ & $\mathrm{X}$ & $\mathrm{X}$ & $\mathrm{X}$ & $\mathrm{X}$ \\
Residencias Grecia & $\mathrm{N} / \mathrm{A}$ & $\mathrm{X}$ & $\mathrm{X}$ & $\mathrm{X}$ & $\mathrm{X}$ & $\mathrm{X}$ \\
Conservatorio de & $\mathrm{N} / \mathrm{A}$ & $\mathrm{X}$ & $\mathrm{X}$ & $\mathrm{X}$ & $\mathrm{X}$ & $\mathrm{N} / \mathrm{A}$ \\
Música & $\mathrm{X}$ & $\mathrm{X}$ & & $\mathrm{X}$ & $\mathrm{X}$ \\
Reserva & $\mathrm{X} / \mathrm{A}$ & $\mathrm{X}$ & $\mathrm{X}$ & $\mathrm{X}$ \\
Museo & $\mathrm{X} / \mathrm{A}$ & $\mathrm{X}$ & $\mathrm{X}$ & $\mathrm{X}$ & $\mathrm{X}$ & $\mathrm{N} / \mathrm{A}$ \\
Centro Infantil & & & & $\mathrm{X}$ \\
\hline
\end{tabular}

Fuente. Morales y Fallas, 2016 
ficios, para aproximar la proporción de excretas que se tratan con tanque séptico o planta de tratamiento. Así pues, se obtuvo de la Oficina de Registro la utilización de las aulas y laboratorios de cada uno de los edificios. Esto dio un porcentaje por recurrencia de 93,07 \% en los edificios con desagüe a la planta de tratamiento y 6,93\% a tanques sépticos.

\section{Residuos sólidos}

Para el análisis de esta fuente, en cada una de las instalaciones de la Sede se realizaron pesajes durante tres semanas que permitieron obtener la cantidad de residuos generados. Luego, con tales datos, se calcularon las emisiones aproximadas del periodo.

\section{$A / C$, refrigerantes y gas}

El dato correspondiente a los rubros de $\mathrm{A} / \mathrm{C}$ y refrigerantes no fue posible calcularlo debido a que el mantenimiento de los equipos de la Sede es realizado por el Departamento de Maquinaria y Equipo de la Sede de San José que no cuenta con un registro de las cargas realizadas por Sede. Con respecto al gas, el Instituto Meteorológico Nacional proporciona un factor de emisión para el gas LP, que fue utilizado tanto en los comedores de la institución como en los laboratorios. En este caso se obtuvo la emisión de $\mathrm{CO} 2$ de acuerdo a la cantidad en litros consumidos durante el 2014.

\section{Electricidad}

El dato de $\mathrm{CO}_{2-\mathrm{e}}$ generado por el consumo de electricidad se obtuvo de los informes de los $\mathrm{kWh}$ mensuales consumidos por la Sede. Los datos fueron brindados por Instituto Costarricense de Electricidad (ICE).

Una vez obtenidos todos los datos, la cuantificación final de las emisiones y el cálculo del $\mathrm{CO}_{2-e^{\prime}}$ por instalación y recinto, se realizó mediante la elaboración de una matriz de cálculo en Excel en la cual se establecieron los datos ne- cesarios para la estimación de las emisiones. En esta hoja se utilizaron dos tipos de fórmulas:

Emisiones por combustible:

$\mathrm{ECO}_{2}=\mathrm{A} * \mathrm{FE}$

Donde:

$\mathrm{ECO}_{2}=$ emisiones $\mathrm{CO}_{2}\left(\mathrm{tCO}_{2} /\right.$ año $)$

$\mathrm{A}=$ datos de actividad (litros/año; kWh/año)

$\mathrm{FE}=$ factor de emisión $\left(\mathrm{tCO}_{2} /\right.$ litro; $\left.\mathrm{tCO}_{2} / \mathrm{kWh}\right)$

Emisiones de otras fuentes de generación:

$$
\mathrm{ECO}_{2-\mathrm{e}}=\mathrm{A} * \mathrm{FE} * \mathrm{PCG}
$$

Donde:

$\mathrm{ECO}_{2-\mathrm{e}}=$ emisiones de $\mathrm{CO}_{2}$ equivalente $\left(\mathrm{tCO}_{2} /\right.$ año)

PCG $=$ potencial de calentamiento global

Los factores de emisión utilizados fueron tomados del IMN. Además, se realizó un análisis $A B C$ para identificar las principales fuentes de emisión de $\mathrm{CO}_{2 e^{\prime}}$ donde:

- Las fuentes con clasificación "A" corresponden a aquellas que generan $80 \%$ de las emisiones.

- Las fuentes con clasificación "B" corresponden a aquellas que generan $15 \%$ de las emisiones.

- Las fuentes con clasificación "C" corresponden a aquellas que generan $5 \%$ de las emisiones.

\section{Resultados y discusión}

\section{Cálculo de $\mathrm{CO}_{2-\mathrm{e}}$ emitido por fuente}

\section{Combustible}

Del total de $\mathrm{CO}_{2}$ emitido por consumo de combustible, $20 \%$ proviene de la utilización de gasolina y el $80 \%$ al diésel. Se consumieron 29 779 litros de combustible, de los cuales $27 \%$ fue gasolina y $73 \%$ diésel. Dicho consumo emite 76,533 Ton CO2, de los cuales 18,482 Ton CO2 son por gasolina y 58,107 Ton CO2, por diésel. 
En la figura 1 se muestra en comportamiento por mes de las emisiones de $\mathrm{CO}_{2}$ generadas por el uso de combustible.

\section{Excretas humanas}

Las emisiones a causa de esta fuente son de aproximadamente 24,13 Ton $\mathrm{CO}_{2-\mathrm{e}^{\mathrm{e}}}$ donde $81 \%$ de estas proviene del tratamiento por tanque séptico y el $19 \%$ de la laguna de tratamiento.

\section{Residuos sólidos}

En la Sede se generaron aproximadamente 29 $450 \mathrm{~kg}$ de residuos. Estos emiten 35,93 Ton $\mathrm{CO}_{2 \text {-e }}$. De ese total, $87 \%$ es emitido por el recinto de San Ramón y 13 \% corresponde al recinto de Grecia.

\section{Gas LP}

La emisión debido a la utilización de gases LP, tanto en laboratorios como en otras oficinas de la Sede, es de aproximadamente 10,01 Ton CO2-e. Durante el año base 2014, la Sede consumió 6920 litros de gas, de los cuales $57 \%$ corresponde al recinto de San Ramón y 43 \% al recinto de Grecia.

\section{Electricidad}

En la figura 2 se muestra el consumo y generación de $\mathrm{CO}_{2-\mathrm{e}}$ mensual para el 2014. Se evidencia una generación total de 120,690 Ton $\mathrm{CO}_{2-e^{\prime}}$ donde 93,91 \% corresponde al recinto de San Ramón y el restante 6,09\% al recinto de Grecia.

En el caso de la Reserva Biológica Alberto Manuel Brenes, se cuenta con una pequeña planta hidroeléctrica, la que genera la energía necesaria para el consumo de las instalaciones, por lo cual no se incluyó en la generación de $\mathrm{CO}_{2-\mathrm{e}}$.

$\mathrm{Al}$ igual que el consumo de combustible, en la figura 2 se destacan picos de emisión al final de cada semestre, lo cual se puede deber a un mayor tiempo de permanencia de los estudiantes en las instalaciones a causa de trabajos y exámenes finales.

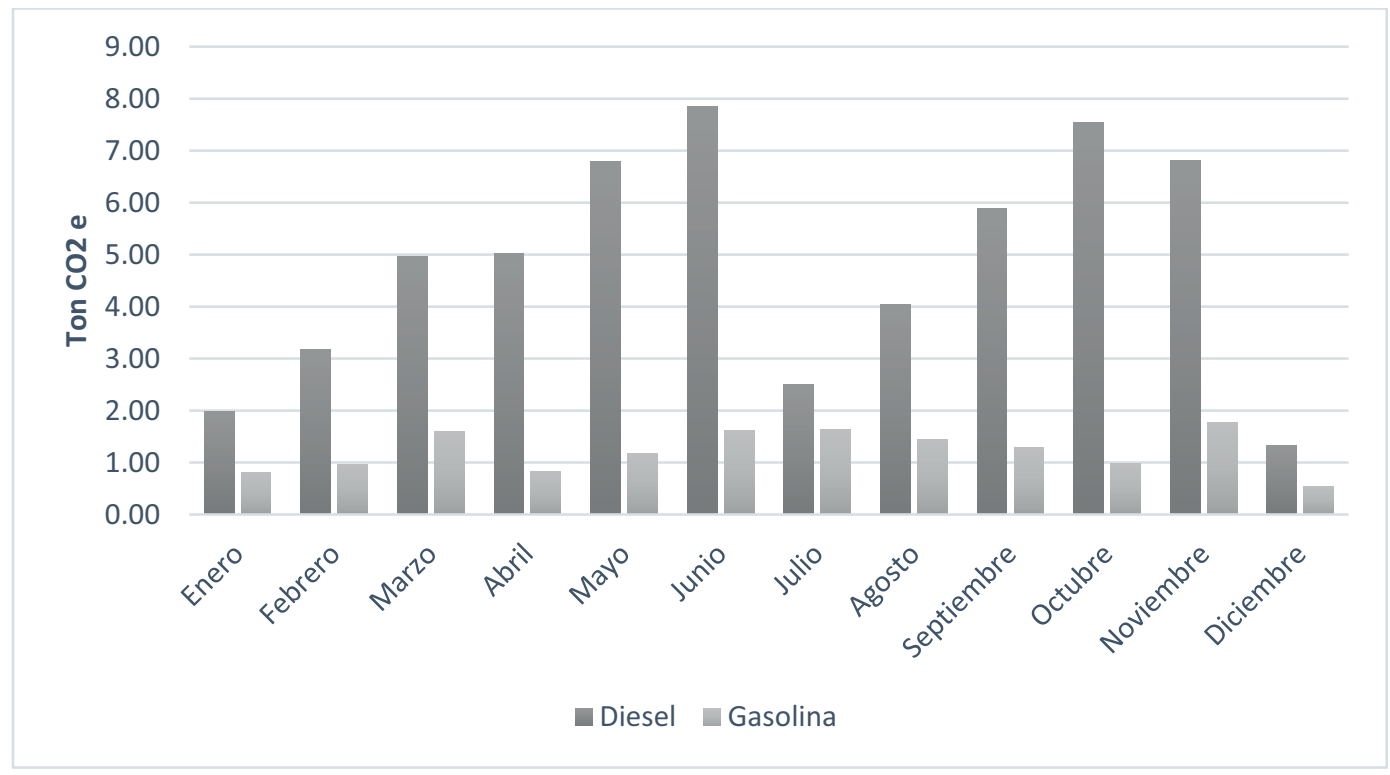

Figura 1. Comportamiento mensual de las emisiones de $\mathrm{CO}_{2 \mathrm{e}}$ generadas por el uso de combustibles. Fuente: Morales y Fallas, 2016. 


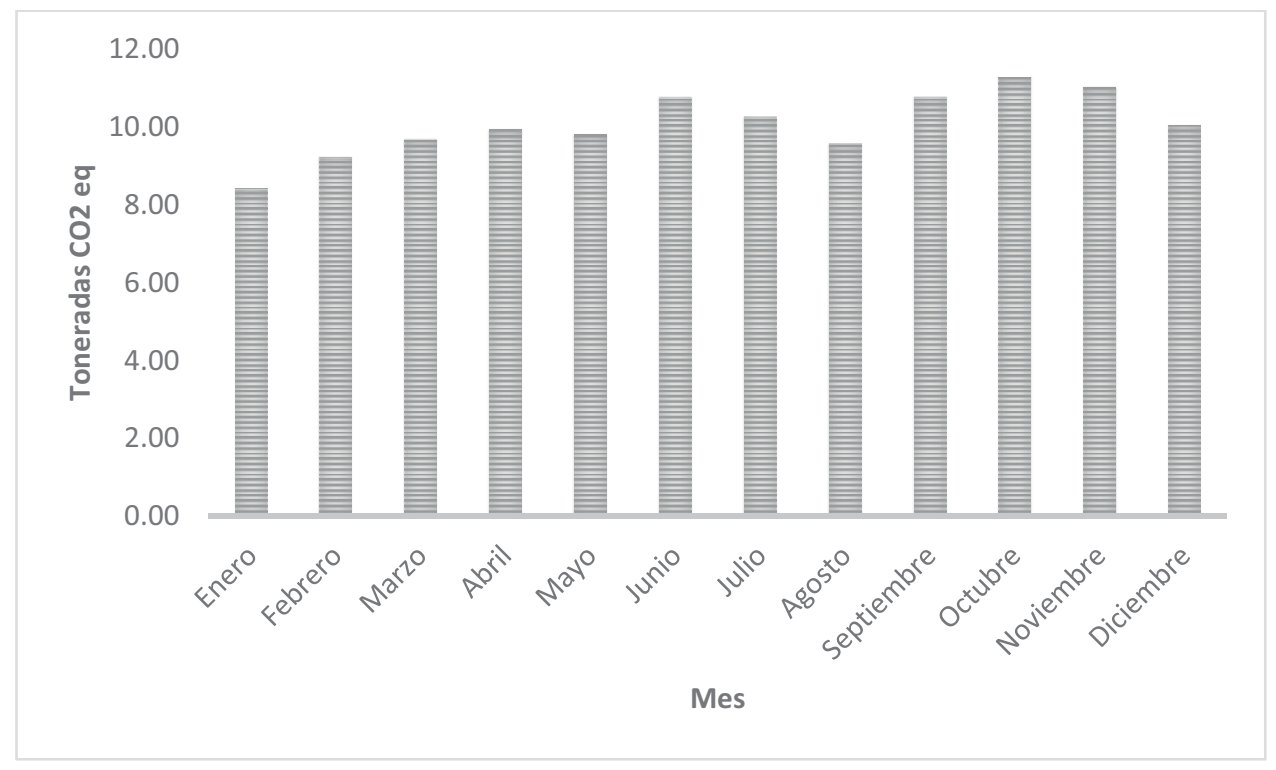

Figura 2. Comportamiento mensual de las emisiones de CO2-e generadas por el consumo de electricidad

\section{Total de emisiones por recinto}

En la tabla 2 se muestra la cantidad de emisiones, en Ton $\mathrm{CO}_{2-\mathrm{e}^{\prime}}$ por fuente y recinto.

La clasificación ABC para las fuentes de emisión, se muestra en la tabla 3. En la tabla 3 se observa que $74 \%$ de las emisiones de $\mathrm{CO}_{2 \text {-e }}$ generadas por las Sede son causadas por el consumo de electricidad y los combustibles. Por lo tanto cualquier estrategia de disminución debe centrarse principalmente en ambos factores o fuentes de emisión, sin dejar de lado las calificadas como B y C.

Tabla 2

Emisiones de la Sede de Occidente por fuente de emisión y Recinto

\begin{tabular}{lccc}
\hline $\begin{array}{c}\text { Fuente de } \\
\text { emisión }\end{array}$ & $\begin{array}{c}\text { Recinto San } \\
\text { Ramón } \\
\text { (ton } \mathrm{CO}_{2-\mathrm{e}} \text { ) }\end{array}$ & $\begin{array}{c}\text { Recinto } \\
\text { Grecia } \\
\text { (ton } \mathrm{CO}_{2-\mathrm{e}} \text { ) }\end{array}$ & $\begin{array}{c}\text { Total Sede } \\
\text { Occidente } \\
\text { (ton } \mathrm{CO}_{2-\mathrm{e}} \text { ) }\end{array}$ \\
\hline Electricidad & 113,33 & 7,36 & 120,69 \\
Combustibles & 72,55 & 4,04 & 76,59 \\
Residuos & 31,37 & 4,56 & 35,93 \\
Excretas & 24,13 & 9 & 24,13 \\
Gases & 6,35 & 3,66 & 10,01 \\
Total & 247,74 & 19,61 & 267,35 \\
Porcentaje & 93 & 7 & 100 \\
\hline
\end{tabular}

Fuente. Morales y Fallas, 2016
Tabla 3

Clasificación $A B C$ de fuentes de emisión

\begin{tabular}{lcccc}
\hline $\begin{array}{c}\text { Fuente de } \\
\text { emisión }\end{array}$ & $\begin{array}{c}\text { Total } \\
\left(\text { Ton } \mathrm{CO}_{2-\mathrm{e}}\right)\end{array}$ & $\%$ & $\begin{array}{c}\% \\
\text { Acumulado }\end{array}$ & Clasificación \\
\hline Electricidad & 120,69 & 45 & 45 & $\mathrm{~A}$ \\
Combustibles & 76,59 & 29 & 74 & $\mathrm{~A}$ \\
Residuos & 35,93 & 13 & 87 & $\mathrm{~B}$ \\
Excretas & 24,13 & 9 & 96 & $\mathrm{C}$ \\
Gases & 10,01 & 4 & 100 & $\mathrm{C}$ \\
\hline
\end{tabular}

Fuente. Morales y Fallas, 2016 


\section{Conclusión}

Por una parte, con los datos recolectados para el año base 2014 fue posible calcular el $\mathrm{CO}_{2 \text {-e }}$ generado por la Sede. Sin embargo, se evidenció que se carece de formas de registro y documentación de datos relevantes para efectuar el cálculo. Asimismo, existe un alto grado de desconocimiento de funcionarios, profesores y estudiantes sobre el tema, lo que provoca menos conciencia de la situación y mayores dificultades para la consecución de la carbono neutralidad.

Por otra parte, es importante rescatar el interés de la Dirección y de la Coordinación de Administración, así como de algunos docentes y estudiantes en realizar algunos cambios en el funcionamiento de la institución, de modo que en los periodos siguientes se logre reducir significativamente la huella generada. Entre los cambios analizados se pueden mencionar el estudio realizado sobre la posible utilización de biodiesel como una alternativa al uso del diésel, la instalación de paneles solares para disminuir el consumo de electricidad brindado por el Instituto Costarricense de Electricidad, la mejora de la planta de tratamiento y la presentación al Ministerio de Salud del Plan de manejo de residuos sólidos.

Pero para que la Sede logre la carbono neutralidad se debe diseñar una herramienta que permita registrar los datos de fuentes directas e indirectas de emisión de gases de efecto invernadero y calcular las emisiones de $\mathrm{CO}_{2-\mathrm{e}}$ generadas. Además, es necesario establecer e implantar una estrategia que permita mitigar los gases generados, la cual debe establecer actividades para la reducción y remoción de las emisiones de $\mathrm{CO}_{2}$. También, para que esta estrategia sea exitosa se debe basar en la obtención de competencias de todos los involucrados de modo que sea posible brindar conocimientos, mejorar habilidades y cambiar actitudes en la población universitaria de la Sede.

\section{Referencias}

Instituto de Normas Técnicas de Costa Rica. (2006). Norma INTE-ISO 14064-1 Gases de Efecto Invernadero Parte 1, 2 y 3. San José, Costa Rica.

Instituto de Normas Técnicas de Costa Rica. (2013). Norma INTE 12-01-06:2011 Norma nacional para demostrar la C-neutralidad. San José, Costa Rica.

Instituto Meteorológico Nacional. (2014). Inventario de gases de efecto invernadero. Recuperado de http://cglobal.imn.ac.cr/gases-efecto-invernadero

Intergovernmental Panel on Climate Change (2001). Cambio Climático 2001: Mitigación. Recuperado de http://www.ipcc.ch/pdf/ climate-changes-2001/mitigation/mitigation-spm-ts-sp.pdf

Ministerio de Ambiente, Energía y Telecomunicaciones. (2009). Estrategia Nacional de Cambio Climático. Recuperado de: http://cambioclimaticocr.com/2012-05-22-19-42-06/estrategia-nacional-de-cambio-climatico

Morales, M. y Fallas, M. (2016). Desarrollo de una estrategia de cuantificación y mitigación de los Gases de Efecto Invernadero (GEI) en la Universidad de Costa Rica Sede de Occidente que permita alcanzar la Carbono Neutralidad (tesis de grado). Sede de Occidente, Universidad de Costa Rica, Costa Rica.

Naciones Unidas (1992). Convención Marco de la Naciones Unidas sobre el Cambio Climático. New York: Naciones Unidas.

Protocolo de Kyoto de la Convención Marco de las Naciones Unidas sobre el Cambio Climático (1998). Recuperado de http://unfccc.int/ resource/docs/convkp/kpspan.pdf

Rodríguez, A., Campos, L.; Ramírez, N.; Mora, R.; y Ramírez, S. (2014). Análisis de capacidad 
y diseño del tercer nivel de la Biblioteca Arturo Agüero Chaves. Trabajo del curso de Localización y Distribución de Instalaciones, Ingeniería Industrial, Sede de Occidente, UCR.

Universidad de Costa Rica (2011). Acta de sesión 5539 del Consejo Universitario. Recuperado de http://cu.ucr.ac.cr/actas/2011/5539.pdf

Universidad de Costa Rica (2017). Sistema de aplicaciones estudiantiles.
Universidad EARTH (2014). ¿Qué es Carbono Neutro? Recuperado de http://www.earth.ac.cr/es/ about-earth/carbono-neutro/spanish-que-es-carbono-neutro/

World Business Council for Sustainable Development, World Resource Institute y Secretaria de Medio Ambiente y Recursos Naturales (2005). Protocolo de Gases Efecto Invernadero. Estándar Corporativo de Contabilidad y Reporte. México.

Recibido: 20 de mayo de 2017 Aceptado: 9 de junio de 2017 